\title{
Do Artificial Neural Networks Always Provide High Prediction Performance? An Experimental Study on the Insufficiency of Artificial Neural Networks in Capacitance Prediction of the 6H-SiC/MEH-PPV/AI diode
}

Andaç Batur Çolak ( $\nabla$ andacbaturcolak@hotmail.com )

Niğde Ömer Halisdemir University: Nigde Omer Halisdemir Universitesi https://orcid.org/0000-00019297-8134

\section{Tamer Güzel}

Niğde Ömer Halisdemir University: Nigde Omer Halisdemir Universitesi

\section{Research Article}

Keywords: Artificial Neural Network, MEH-PPV, capacitance-voltage, Schottky diode, barrier height

Posted Date: October 29th, 2021

DOI: https://doi.org/10.21203/rs.3.rs-1019177/v1

License: (c) (i) This work is licensed under a Creative Commons Attribution 4.0 International License. Read Full License 


\section{Abstract}

Recently, studies on artificial neural network model, which is one of the most effective artificial intelligence tools applied in many fields, reported that artificial neural networks are tools that offer very high prediction performance compared to traditional models. In this study, an artificial neural network model has been developed to predict the capacitance voltage outputs of the $6 \mathrm{H}-\mathrm{SiC} / \mathrm{MEH}-\mathrm{PPV} / \mathrm{Al}$ diode with organic polymer interface, depending on the frequency. In the multi-layer network model developed with a total of 186 experimental data, $70 \%$ of the data used for training, $15 \%$ for validation and $15 \%$ for testing. The prediction performances of three different artificial neural networks developed with 5, 10 and 15 neurons in their hidden layers have been analyzed. The results obtained, for the first time in the literature, show that the artificial neural network model cannot predict the capacitance voltage outputs of the organic polymer interface $6 \mathrm{H}-\mathrm{SiC} / \mathrm{MEH}-\mathrm{PPV} / \mathrm{Al}$ diode depending on the frequency.

\section{Introduction}

Artificial neural network systems (ANN) have become the subject of many researchers and studies today due to its capabilities. In addition to the ongoing studies on the limits and potential of the ANN system, its application areas continue to increase day by day. The ANN system basically works in a similar way to the working principle of neurons [1]. There are artificial neurons that imitation nerve cells. These neurons generally act as a multi-input and single-output nonlinear element [2]. They form multi-layer perceptron neural networks by creating connections between them. With these layers, outputs are obtained depending on the algorithm used after a process similar to the learning process [3]. This unique functioning of the ANN system has offered them the opportunity to use in many areas. In particular, the ability of these systems to create artificial data, as well as their success in predicting non-linear data has opened up many application areas from health to energy and electronics [4-8]. One of these areas is metal semiconductor contacts [9]. Metal-semiconductor contacts are one of the milestones in the development of electronics science since the day they were produced. A physical barrier between metal and semiconductor has provided humanity with great opportunities in the control of electrons [10]. Many electronic circuit elements have been developed based on this structure known as the Schottky barrier [11-13]. The most important of these are diodes. Diodes are used in many areas of electronics today. One of these diodes are Schottky diodes based on silicon carbide (SiC) $[14,15]$. These diodes differ from conventional diodes with their ability to operate in extreme conditions $[16,17]$. The area of use of diodes is directly proportional to their electrical characteristics. Therefore, the ANN system may have the potential to play an important role in determining the electrical parameters of these diodes. At this point, it is very important to investigate the usability of the ANN system in this field or to determine the limits of its potential.

According to the literature, the applications of the ANN system on Schottky diodes seem quite successful due to the analytical data obtained from diode measurements. Lim et al. tested the inference accuracy of the gated Schottky diodes by constructing an ANN neural network. They reported that the ANN system can be classify with a very high accuracy when the outputs obtained from the artificial neural network are 
compared with the outputs obtained from test devices [18]. Rabehi et al. [19] presented a new approach to estimate the Schottky diode parameters with greater accuracy. They demonstrated that the parameters of the Schottky diode can be successfully determined using a new function algorithm. Mellit et al. [20] proposed a simple ANN model for modeling and estimating the power generated by the photovoltaic module consisting of polycrystalline Schottky diodes. They explained that the proposed model can predict module output power with acceptable accuracy. M. Alade [21] used ANN system to calculate the electrical parameters of GaN Schottky diodes at high temperatures. He compared the outputs of this system with theoretical calculation results. He showed that the calculated results and the theoretical results are in good agreement. Darwish et al. [22] declared an efficient approach to calculate currentvoltage outputs using the ANN system. They explained that the simulation results resulting from the ANN system show a clear and perfect match with the experimental data. They proved that the ANN approach can be effectively used to model the current-voltage at the $p-n$ junction. Mittal et al. [23] investigated the usability of the ANN system in examining the performance of photovoltaic Schottky diodes. They created a network model because current-voltage is output for this. They compared the experimental photovoltaic diode output with the ANN system output and the data they obtained and showed that the results were in great consistency. Liang et al. [24] investigated the availability of the ANN system in the design of shottky barrier diodes. They analyzed the nonlinear power properties of the diode according to the ANN model. According to this study, they stated that the experimental parameters and ANN data were in good agreement.

Çolak [25] developed an ANN model to predict the viscosity of five different concentrations of $\mathrm{ZrO}_{2} /$ Water nanofluid at different temperatures. The developed MLP network was able to predict the viscosity of the nanofluid with high accuracy depending on the temperature and concentration. Rahman et al. [26] applied an ANN model to estimate the oscillating heat transfer coefficient of a thermoacoustic heat exchanger. The ANN model developed for the oscillating heat exchanger used in a floating thermoacoustic refrigerator was developed using experimental data. Two input parameters are used in the MLP network, which has a hidden layer containing ten optimal neurons. The results obtained confirmed that the ANN model has high accuracy prediction performance. Colak et al. [27] studied the development of an ANN model to predict the effect of high temperature on the flexural and compressive strength of mortars containing waste PET aggregates. In the ANN model developed using experimental data, bending and compressive strengths are simulated depending on the temperature and concentration. As a result of the study, it was seen that the ANN model can predict bending and compressive strength values with very low error rates. Pang et al. [28] worked on the development of an ANN model for predicting solar radiation. Real meteorological data from a local weather station were used to train the ANN model. The data obtained from the designed ANN model were compared with the target data and the accuracy of the model was analyzed. The findings show that the ANN model can provide predictive data with high agreement with the target data. Çolak [29] developed an ANN application to predict the spread of the COVID-19 virus using published data from different countries. The simulation results obtained from the developed MLP model were compared with the target data and the performance 
analysis of the network was made. The performance analysis results of the ANN model have shown that the simulation results are in ideal agreement with the target data.

As can be seen from the extensive literature review, it is stated that ANNs have high prediction performance in all studies reported in the literature. In this study, an application where an ANN model failed has been reported for the first time. The ANN model, which has been developed to predict the capacitance voltage outputs of the $6 \mathrm{H}-\mathrm{SiC} / \mathrm{MEH}-\mathrm{PPV} / \mathrm{Al}$ diode with organic polymer interface, depending on the frequency, showed extremely high error rates and failed to predict the target data. In all the studies in the literature, the root causes of the failure of the ANN model, which is stated to be a mathematical tool with very high prediction performance, have been extensively examined and discussed. This study, in which the failure of the ANN model has been reported for the first time in the literature, is of great importance because it fills many gaps and is the first study reported on this subject in the literature.

\section{Experimental Model}

6H-SiC/MEH-PPV/AI Schottky barrier diode is fabricated by Cree Inc. A $280 \mu \mathrm{m}$ thick n-type $6 \mathrm{H}-\mathrm{SiC}$ wafer, with a diameter of 2 inches (001), with a donor density of $2.61017 \mathrm{~cm}-3$, was used. The surface of the SiC wafer was cleaned using the clean method known as RCA. Then, it was kept in HF / H20 (1:10) solution for 20 seconds to remove the oxide layer formed on the surface. After this process, pure gold $(99.995 \%)$ of $150 \mathrm{~nm}$ thickness was evaporated to the surface of the SiC wafer under 10-6 Torr pressure. To compose ohmic contact, the sample was annealed at $500^{\circ} \mathrm{C}$ for 5 minutes in the metal evaporation system. Then dissolved in toluene, MEH-PPV was coated with a spin coater on the surface of the SiC wafer at $2000 \mathrm{rpm}$ for 60 seconds. The coated $\mathrm{SiC}$ was annealed at $60^{\circ} \mathrm{C}$ for 5 minutes to remove toluene from the wafer surface. After this process, pure aluminum (99.999\%) of $140 \mathrm{~nm}$ thickness was evaporated to form rectifier(Schottky) contact. The schematic diagram of the experimentally produced $6 \mathrm{H}-\mathrm{SiC} / \mathrm{MEH}-\mathrm{PPV} / \mathrm{Al}$ Schottky diode is shown in Figure 1. Keithley 2400 Sourcemeter was used for current-voltage (I-V) measurements. Measurements were carried out with the help of an IEEE-488 AC / DC converter card installed in the computer. Current-Voltage $(\mathrm{I}-\mathrm{V})$ was done in the voltage range of $-3 \mathrm{~V}$ to + $3 \mathrm{~V}$.

\section{Ann Model Development}

In the literature, there are various mathematical tools used by researchers for data processing and modeling purposes [30-34]. Traditional methods used in creating simulation models using data; It can be seen that time-dependent data are insufficient in some cases such as irregularity and modeling of nonlinear functions [35]. Recently, artificial intelligence (AI) based prediction models have become tools that are frequently used by researchers in many areas. Al-based forecasting is based on simulation modeling after training the system using data. Artificial neural networks (ANN) are one of the Al tools popularly used by researchers [36]. In the literature, the ANN models; It is stated that it has advantages such as straight routes in the learning phase of nonlinear correlations, the ability to move together analogue and digital data, the convenience of reusing information thanks to feedback, its strong structure 
even in the presence of noisy input data, and high sensitivity in learning new data [37]. There are ANN models with different structures such as radial basic functional neural network (RBFNN) [38], convolutional neural network (CNN) [39] and multi-layer perceptron (MLP) [40]. Among these models, the frequently preferred model is the MLP network model. While MLP networks have an input layer and an output layer, there is at least one hidden layer. In the hidden layer of the MLP network are computing units called neurons. Each link between layers has a weight associated with it. Signals passing through each link between layers are multiplied by the respective weight. A bias is added by summing the inputs from the neurons in the next layers, and the result is reached by using a transfer function to obtain the value in the output layer. In an MLP network with a feed-forward (FF) back-propagation (BP) approach, a match is created between nonlinear rules derived from input data and output data. Training of the MLP network in the FF stage is carried out by updating biases and weights. Data from the input layer is feed-forward and by optimizing the weights between neurons, back-propagation of errors is achieved during the training phase. The training phase of the ANN model continues in this way until the lowest error rate is reached and the training of the ANN model is terminated when the lowest error value is reached.

In this study, an ANN model has been developed to predict the capacitance voltage outputs of an organic polymer interface $6 \mathrm{H}-\mathrm{SiC} / \mathrm{MEH}-\mathrm{PPV} / \mathrm{Al}$ diode depending on the frequency. The voltage $(\mathrm{V})$ and frequency (F) values in the input layer of the MLP network model, which has been developed with a total of 186 data, have been defined as input parameters and the capacitance (C) value has been predicted at the output layer. Optimizing the data used in training ANNs is one of the important parameters that directly affect the prediction performance of ANN [41]. For this reason, optimum data grouping has been preferred by making different data optimizations. The data used in the development of ANN are grouped in three separate sections as frequently preferred in the literature [42-44]. $70 \%$ of the dataset has been used for training, $15 \%$ for validation and $15 \%$ for testing. The basic configuration structure of the developed ANN model is shown in Figure 2.

The number of neurons used in the hidden layers of ANNs is one of the important parameters affecting the prediction performance of ANNs. There is no accepted modeling or calculation tool for determining the number of neurons to be used in ANN models $[45,46]$. For this reason, three different ANN models with 5, 10 and 15 neurons in the hidden layers have been developed to consider the performance of the models developed with different neuron numbers. In the developed ANN model, the Levenberg-Marquardt algorithm, one of the powerful algorithms used frequently by researchers, has been used as the training algorithm [47]. In the hidden layer of MLP network, Tan-Sig function is used as the transfer function and Purelin functions in the output layer [48]. The transfer functions used are given below:

$$
f(x)=\frac{1}{1+\exp (-x)}(1)
$$

$\operatorname{purelin}(\mathrm{x})=\mathrm{x}(2)$

Some parameters should be examined in order to analyze the prediction performance of the developed ANN model. For this purpose, the Mean Square Error (MSE), Coefficient of Determination (R) parameters 
have been used to evaluate the performance of the MLP network. The deviation rate between the predicted values obtained from the ANN model and the target values has also been analyzed. The equations used in the calculation of performance parameters are given below [49].

$$
\begin{gathered}
\text { MSE }=\frac{1}{\mathrm{~N}} \sum_{\mathrm{i}=1}^{\mathrm{N}}\left(\mathrm{X}_{\operatorname{exp~(i)}}-\mathrm{X}_{\mathrm{ANN}(\mathrm{i})}\right)^{2}(3) \\
\mathrm{R}=\sqrt{1-\frac{\sum_{\mathrm{i}=1}^{\mathrm{N}}\left(\mathrm{X}_{\operatorname{exp~(i)}}-\mathrm{X}_{\mathrm{ANN}(\mathrm{i})}\right)^{2}}{\sum_{\mathrm{i}=1}^{\mathrm{N}}\left(\mathrm{X}_{\operatorname{exp~(i)}}\right)^{2}}}(4) \\
\text { ErrorRate }(\backslash \%)=\left[\frac{\mathrm{X}_{\exp }-\mathrm{X}_{\mathrm{ANN}}}{\mathrm{X}_{\exp }}\right] \mathrm{x} 100(5)
\end{gathered}
$$

\section{Results And Discussion}

Capacitance-voltage graph of the $6 \mathrm{H}-\mathrm{SiC} / \mathrm{MEH}-\mathrm{PPV} / \mathrm{Al}$ diode at a frequency range of $50-1000 \mathrm{kHz}$ and at room temperature is shown in Figure 3. When Figure 3 is examined, it can be seen that as the frequency increases, the capacitance value also increases. However, according to the literature, it is reported that as the frequency increases, the capacitance decreases [50]. This may be due to the MEH-PPV polymer present at the interface. Because the conductive polymer layer at the interface can affect current conduction. This may have affected the measured value of the diode capacitance. Reddy [51] showed that the conductive polymer layer at the interface can affect the capacitance value of the diode.

Figure 4, Figure 5 and Figure 6 show graphs of the data obtained for each stage of the ANN models developed with 5, 10 and 15 neurons, respectively. While there are target values on the x-axes of the graphs, there are ANN predictions on the $y$-axes. The values indicated by the dotted line are equality line and those indicated by solid color lines are the fitted data values. The purpose of these graphs is to analyze and show the proximity of ANN outputs to the target values and the error rates between them. When the studies on ANN in the literature are examined, it is seen that ANN is a powerful mathematical tool that can make predictions with high accuracy. In the figures obtained from these studies, it is seen that the fitted data and the equality line are very close and compatible with each other, however, the data points are located on or very close to both lines. When the equality line, fitted line and data points shown in graphics regarding the data obtained from each stage of the ANN model are carefully examined, the discrepancy between the data is clearly seen. It should be noted that in each of the graphics, equality line and fitted line trends are opposite to each other. However, the distance and mismatch of data points from both lines is also clearly visible. Analyzing the R values calculated for the ANN model is important in terms of seeing the prediction accuracy of the ANN. The proximity of the R values to the 1 value is directly proportional to the prediction accuracy of the ANN model. When the studies conducted on ANN by the researchers in the literature are examined, it is seen that the $\mathrm{R}$ values are very close to 1 and positive 
values. When the $\mathrm{R}$ values calculated for each stage of the ANN model developed in this study are examined, it is seen that a situation that is not similar to the situation in the literature has emerged. When the $R$ values obtained from each developed ANN model are examined, the distances of the values from 1 are seen. The numerical values of the $R$ values show that the prediction performance of each ANN model is very low and it can make predictions with high error.

In Figure 7, experimental data and ANN predictions are shown on the same graph. While there are voltage values on the $x$-axes of the graphs, there are capacitance values on the $y$-axes. In the graphs shown separately for each frequency value, the changes of the capacitance values at the fixed frequency with respect to the voltage are given. The purpose of presenting these graphs is to enable the discrepancy between ANN predicts and target values to be seen more clearly. When similar figures are examined in the literature, it is seen that target values and ANN estimation data are located on or very close to each other. The proximity of ANN predictions to target values indicates that the ANN model makes predictions with high accuracy. When Figure 7 is examined, it is seen that the data points obtained from the ANN model and the target data points are incompatible and located at very different points. However, when the trends of data lines are indexed, there are also obvious differences and mismatches in trend trends. These data show that the developed ANN model fails to predict the capacitance voltage outputs of the $6 \mathrm{H}-\mathrm{SiC} / \mathrm{MEH}$ PPV/Al diode with organic polymer interface, depending on the frequency.

In Figure 8, the error rates between the data obtained from the ANN model and the target values for each data point are given. When the error rates of ANN models reported in the literature are examined in detail, it is seen that generally low values are obtained and data points are located around the zero error line. The proximity of the data points to the zero error line shows that the developed ANN model can make predictions with low error rates. When Figure 8 is examined, it can be clearly seen that the data points are far from the zero error line. These extremely high error rates show that there are extremely high error rates between the predicted values obtained from the developed ANN model and the target values.

Figure 9 shows the predicted values and target values obtained from ANN model on the same graph. On the $x$-axis of the graph, there are experimental data with target values, while on the $y$-axis there are predictive values obtained from the ANN model. The increase in the proximity of the data points to the equality line drawn in blue means that the error rates of the ANN model decrease. When the data obtained from the studies conducted on ANN models by the researchers in the literature are examined, it is seen that the data points are located around the equality line. The results obtained from these studies show that ANN models can make predictions with high accuracy. However, considering the data obtained from the ANN model developed in this study, it is seen that the location of the data points is unrelated to the equality line. When these data are analyzed, the failure of the ANN model, which has been developed to predict the capacitance voltage outputs of the $6 \mathrm{H}-\mathrm{SiC} / \mathrm{MEH}-\mathrm{PPV} / \mathrm{Al}$ diode with organic polymer interface, depending on the frequency, is clearly seen. Performance parameters calculated for the developed ANN model are given in Table 1. 
Table 1

Performance parameters for the ANN model

\begin{tabular}{|llll|}
\hline Model & MSE & $\mathbf{R}$ & MoD $_{\mathrm{av}}(\%)$ \\
\hline 5 Neuron & $1.39 \mathrm{E}-17$ & 0.09967 & -34.92 \\
\hline 10 Neuron & $3.66 \mathrm{E}-17$ & -0.36140 & -68.64 \\
\hline 15 Neuron & $5.89 \mathrm{E}-16$ & 0.39982 & -114.73 \\
\hline
\end{tabular}

It is considered that there may be two reasons why the developed ANN model fails in this way in predicting the capacitance voltage outputs of the $6 \mathrm{H}-\mathrm{SiC} / \mathrm{MEH}-\mathrm{PPV} / \mathrm{Al}$ diode with organic polymer interface, depending on the frequency. The former may be caused by physical reasons due to the conductive polymer layer used between metal and semiconductor surfaces during its production. Because these polymer layers used at the interface can affect current conduction [52]. This may have caused an irregularity in the charges that create a capacitive effect on the interface. This irregularity in the interface may have affected the connection between capacitance-voltage-frequency. This may have caused the ANN system to make incorrect predictions. The other reason is that the learning algorithm of the ANN model is insufficient to learn the data that is irregular and there is no relationship, even if it is complex.

\section{Conclusions}

In this study, three different ANN models have been developed to predict the capacitance voltage outputs of a $6 \mathrm{H}-\mathrm{SiC} / \mathrm{MEH}-\mathrm{PPV} / \mathrm{Al}$ diode with an organic polymer interface depending on the frequency. Levenberg-Marquardt algorithm has been used as the training algorithm in MLP networks developed with 5,10 and 15 neurons in the hidden layers. 130 of the data used in the ANN models, which have been developed using a total of 186 experimental data, have been used in the training of the model, 28 in the validation phase and 28 in the testing phase. MSE, R and MoD parameters have been analyzed for performance analysis of ANN models. The results show that the ANN models have very high error rates and the developed ANN models cannot predict the capacitance voltage outputs of the organic polymer interface $6 \mathrm{H}-\mathrm{SiC} / \mathrm{MEH}-\mathrm{PPV} / \mathrm{Al}$ diode depending on the frequency.

\section{Declarations}

\section{Declaration of interest}

The authors have no competing interests to declare that are relevant to the content of this article.

\section{References}

[1] W. S. McCulloch, W. Pitts, A logical calculus of the ideas immanent in nervous activity. The bulletin of mathematical biophysics 5 (1943) 115-133. 
[2] S. Ding, H. Li, C. Su, J. Yu, F. Jin, Evolutionary artificial neural networks: a review. Artificial Intelligence Review 39 (2013) 251-260.

[3] R. Tanty, T. S. Desmukh, Application of artificial neural network in hydrology-A review. Int. J. Eng. Technol. Res 4 (2015) 184-188.

[4] Z. Zhang, K. Friedrich, Artificial neural networks applied to polymer composites: a review. Composites Science and technology 63 (2003) 2029-2044.

[5] P. J. Lisboa, A. F. Taktak, The use of artificial neural networks in decision support in cancer: a systematic review. Neural networks 19 (2006) 408-415.

[6] A. K. Yadav, S. Chandel, Solar radiation prediction using Artificial Neural Network techniques: A review. Renewable and sustainable energy reviews 33 (2014) 772-781.

[7] R. Kumar, R. Aggarwal, J. Sharma, Energy analysis of a building using artificial neural network: A review. Energy and Buildings 65 (2013) 352-358.

[8] P. Sharma, T. Bhatti, A review on electrochemical double-layer capacitors. Energy conversion and management 51 (2010) 2901-2912.

[9] A. B. Çolak, T. Güzel, O. Yıldız, M. Özer, An experimental study on determination of the shottky diode current-voltage characteristic depending on temperature with artificial neural network. Physica B: Condensed Matter, (2021) 412852.

[10] E. Rhoderick, R. Williams, Oxford, 1988.

11. Q. Liu, S. Lau, A review of the metal-GaN contact technology. Solid-State Electronics 42, 677-691 (1998).

[12] P. Blom, R. Wolf, J. Cillessen, M. Krijn, Ferroelectric schottky diode. Physical Review Letters 73 (1994) 2107.

[13] V. Rideout, A review of the theory, technology and applications of metal-semiconductor rectifiers. Thin Solid Films 48 (1978) 261-291.

[14] J. H. Zhao, K. Sheng, R. C. Lebron-Velilla, Silicon carbide schottky barrier diode. International journal of high speed electronics and systems 15 (2005) 821-866.

[15] T. Güzel, A. K. Bilgili, M. Özer, Investigation of inhomogeneous barrier height for Au/n-type 6H-SiC Schottky diodes in a wide temperature range. Superlattices and Microstructures 124 (2018) 30-40.

[16] X. She, A. Q. Huang, Ó. Lucía, B. Ozpineci, Review of silicon carbide power devices and their applications. IEEE Transactions on Industrial Electronics 64 (2017) 8193-8205. 
[17] X. Wang, J. Qi, M. Yang, G. Zhang, Analysis of 600 V/650 V SiC schottky diodes at extremely high temperatures. CPSS Transactions on Power Electronics and Applications 5 (2020) 11-17.

[18] S. Lim et al., Highly Reliable Inference System of Neural Networks Using Gated Schottky Diodes. IEEE Journal of the Electron Devices Society 7 (2019) 522-528.

[19] A. Rabehi et al., Optimal estimation of Schottky diode parameters using a novel optimization algorithm: Equilibrium optimizer. Superlattices and Microstructures 146 (2020) 106665.

[20] A. Mellit, S. Sağlam, S. A. Kalogirou, Artifıcial neural network-based model for estimating the produced power of a photovoltaic module. Renewable Energy 60 (2013) 71-78.

[21] M. O. Alade, High Temperature Electronic Properties of a Microwave Frequency Sensor-GaN Schottky Diode. Adv. Phys. Theor. Appl 15 (2013) 47-53.

[22] A. Darwish et al., Optoelectronic performance and artificial neural networks (ANNs) modeling of nInSe/p-Si solar cell. Superlattices and Microstructures 83 (2015) 299-309.

[23] M. Mittal, B. Bora, S. Saxena, A. M. Gaur, Performance prediction of PV module using electrical equivalent model and artificial neural network. Solar Energy 176 (2018) 104-117.

[24] A. Liang, Y. Xu, S. Jia, G. Sun, in 2008 International Conference on Microwave and Millimeter Wave Technology. IEEE 2, (2008) 558-561.

[25] A.B. Çolak, A novel comparative analysis between the experimental and numeric methods on viscosity of zirconium oxide nanofluid: Developing optimal artificial neural network and new mathematical model, Powder Technology 381 (2021) 338 - 351.

[26] A.A. Rahman, X. Zhang, Prediction of oscillatory heat transfer coefficient for a thermoacoustic heat exchanger through artificial neural network technique, International Journal of Heat and Mass Transfer 124 (2018) $1088-1096$.

[27] A.B. Çolak, K. Akçaözoğlu, S. Akçaözoğlu, G. Beller, Artifıcial intelligence approach in predicting the effect of elevated temperature on the mechanical properties of PET aggregate mortars: An experimental study, Arabian Journal for Science and Engineering 46 (2021) 4867 - 4881.

[28] Z. Pang, F. Niu, Z. O’Neill, Solar radiation prediction using recurrent neural network and artificial neural network: A case study with comparisons, Renewable Energy 156 (2020) 279 - 289.

[29] A.B. Çolak, Prediction of infection and death ratio of CoVID-19 virus in Turkey by using artificial neural network (ANN), Coronaviruses 2:1 (2021) $106-112$.

[30] W. Gao, J. L.G. Guirao, B. Basavanagoud, J. Wu, Partial multi-dividing ontology learning algorithm, Inf. Sci. 467 (2018) 35-58. 
[31] W. Gao, W. Wang, D. Dimitrov, Y. Wang, Nano properties analysis via fourth multiplicative ABC indicator calculating, Arab. J. Chem. 11 (2018) 793-801.

[32] W. Gao, H. Wu, M.K. Siddiqui, A.Q. Baig, Study of biological networks using graph theory, Saudi J. Biol. Sci. 25 (2018) 1212-1219.

[33] W. Gao, J.L.G. Guirao, M. Abdel-Aty, W. Xi, An independent set degree condition for fractional critical deleted graphs, Dis. Cont. Dyn. Syst.-S 12 (2019) 877-886.

[34] W. Gao, D. Dimitrov, H. Abdo, Tight independent set neighborhood union condition for fractional critical deleted graphs and ID deleted graphs, Dis. Cont. Dyn. Syst.-S 12 (2019) 711-721.

[35] E. Bas, V.R. Uslu, E. Egrioglu, Robust learning algorithm for multiplicative neuron model artificial neural networks, Expert Systems With Applications 56 (2016) 80-88.

[36] M. Vakili, S.Khosrojerdi, P. Aghajannezhad, M.Yahyaei, A hybrid artificial neural network-genetic algorithm modeling approach for viscosity estimation of graphene nanoplatelets nanofluid using experimental data, International Communications in Heat and Mass Transfer 82 (2017) 40-48.

[37] M. Bahiraei, S. Heshmatian, H. Moayedi, Artificial intelligence in the field of nanofluids: A review on applications and potential future directions, Powder Technology 353 (2019) 276-301.

[38] J. Park, I.W. Sandberg, Universal approximation using radial-basis-function networks, Neural Comput. 3 (1991) 246-257.

[39] M.V. Valueva, N.N. Nagornov, P.A. Lyakhov, G.V. Valuev, N.I. Chervyakov, Application of the residue number system to reduce hardware costs of the convolutional neural network implementation, Math. Comp. Simul. 177 (2020) 232-243.

[40] S. Garg, A.M. Shariff, M.S. Shaikh, B. Lal, H. Suleman, N. Faiqa, Experimental data, thermodynamic and neural network modeling of $\mathrm{CO} 2$ solubility in aqueous sodium salt of I-phenylalanine, J. CO2 Utilization 19 (2017) 146-156.

[41] A.B. Çolak, An experimental study on the comparative analysis of the effect of the number of data on the error rates of artificial neural networks, International Journal of Energy Research, 45(1) (2021) 478 500 .

[42] A. Barati-Harooni, A. Najafi-Marghmaleki, An accurate RBF-NN model for estimation of

Viscosity of nanofluids, J. Mol. Liq. 224 (2016) 580-588.

[43] S.H. Rostamian, M. Biglari, S. Saedodin, M.H. Esfe, An inspection of thermal conductivity of CuOSWCNTs hybrid nanofluid versus temperature and concentration using experimental data, ANN modeling and new correlation, J. Mol. Liq. 231 (2017) 364-369. 
[44] F. Esmaeilzadeh, A.S. Teja, A. Bakhtyari, The thermal conductivity, viscosity, and cloud points of bentonite nanofluids with n-pentadecane as the base fluid, J. Mol. Liq. 300 (2020) 112307.

[45] H. Bonakdari, A.H. Zaji, Open channel junction velocity prediction by using a hybrid self-neuron adjustable artificial neural network, Flow Measurement and Instrumentation 49 (2016) 46-51.

[46] A.B. Çolak, Developing optimal artificial neural network (ANN) to predict the specific heat of water based yttrium oxide (Y2O3) nanofluid according to the experimental data and proposing new correlation, Heat Transfer Research, 51(17) (2020) 1565 - 1586.

[47] E. Ahmadloo, S. Azizi, Prediction of thermal conductivity of various nanofluids using artificial neural network, International Communications in Heat and Mass Transfer 74 (2016) 69-75.

[48] A.B. Çolak, Experimental study for thermal conductivity of water-based zirconium oxide nanofluid: Developing optimal artificial neural network and proposing new correlation, International Journal of Energy Research, 45(2) (2020) 2912 - 2930.

[49] A.B. Çolak, O. Yıldız, M. Bayrak, B.S. Tezekici, Experimental study for predicting the specifıc heat of water based $\mathrm{Cu}-\mathrm{Al} 2 \mathrm{O} 3$ hybrid nanofluid using artificial neural network and proposing new correlation, International Journal of Energy Research 44(9), (2020) 7198-7215.

[50] B. Gunduz, I. Yahia, F. Yakuphanoglu, Electrical and photoconductivity properties of p-Si/P3HT/Al and p-Si/P3HT: MEH-PPV/Al organic devices: Comparison study. Microelectronic Engineering 98 (2012) 4157.

[51] V. R. Reddy, Electrical properties of Au/polyvinylidene fluoride/n-InP Schottky diode with polymer interlayer. Thin Solid Films 556 (2014) 300-306.

[52] S. Forrest, P. Schmidt, Semiconductor analysis using organic-on-inorganic contact barriers. I. Theory of the effects of surface states on diode potential and ac admittance. Journal of applied physics 59 (1986) 513-525.

\section{Figures}




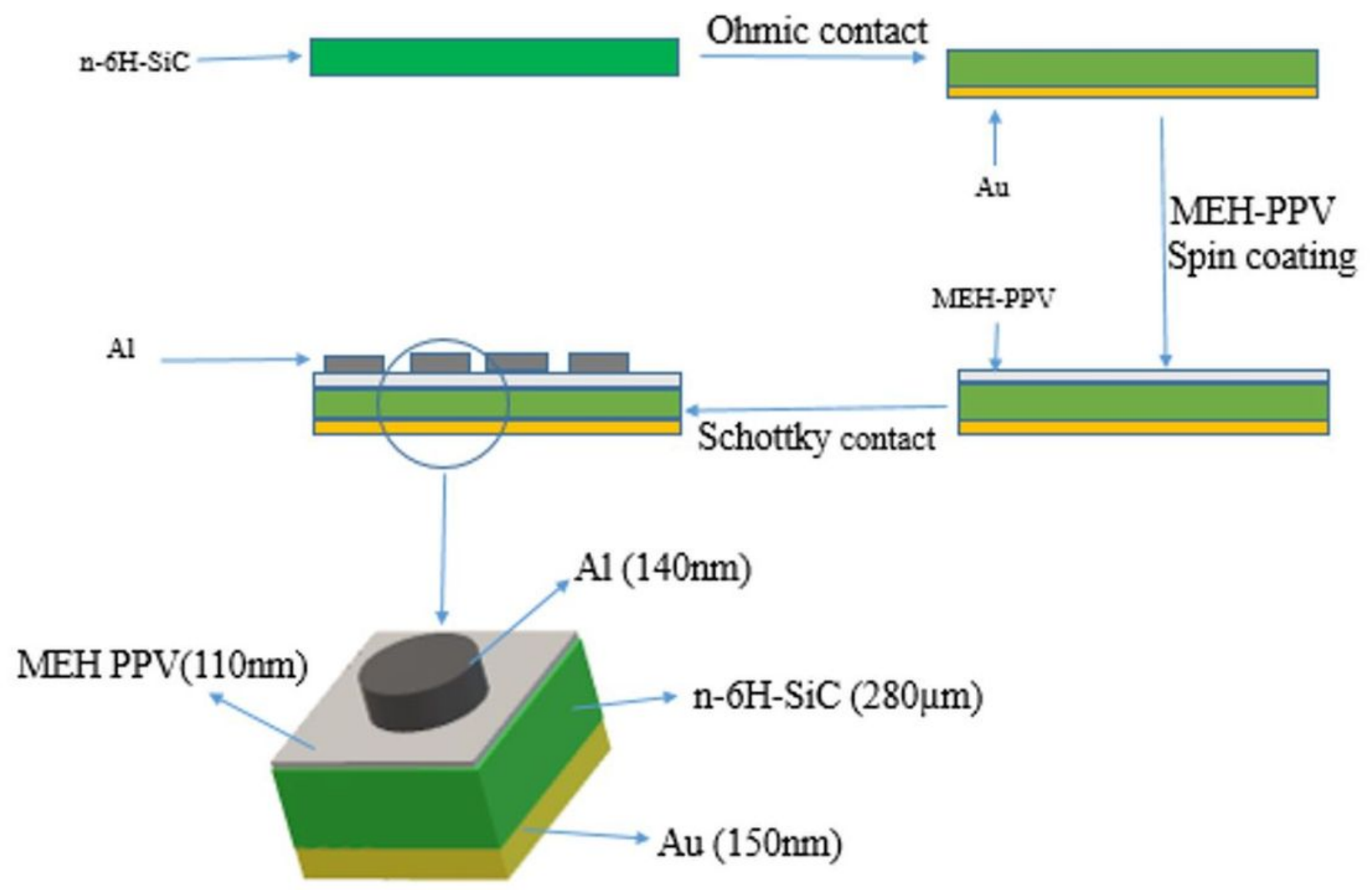

Figure 1

Schematic diagram of $6 \mathrm{H}-\mathrm{SiC} / \mathrm{MEH}$ PPV/Al Schottky diode 

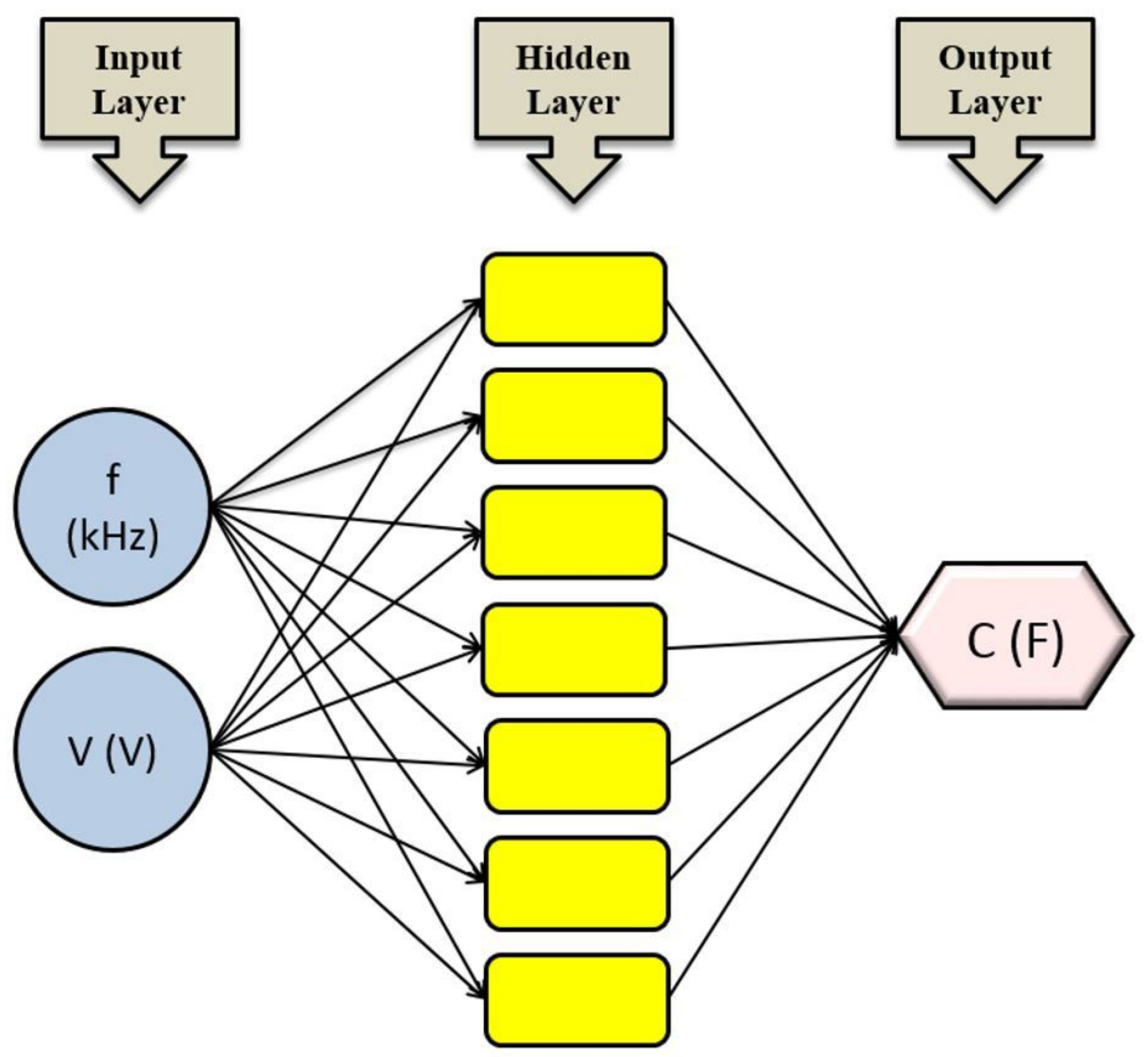

Figure 2

The basic configuration structure of the developed ANN model 


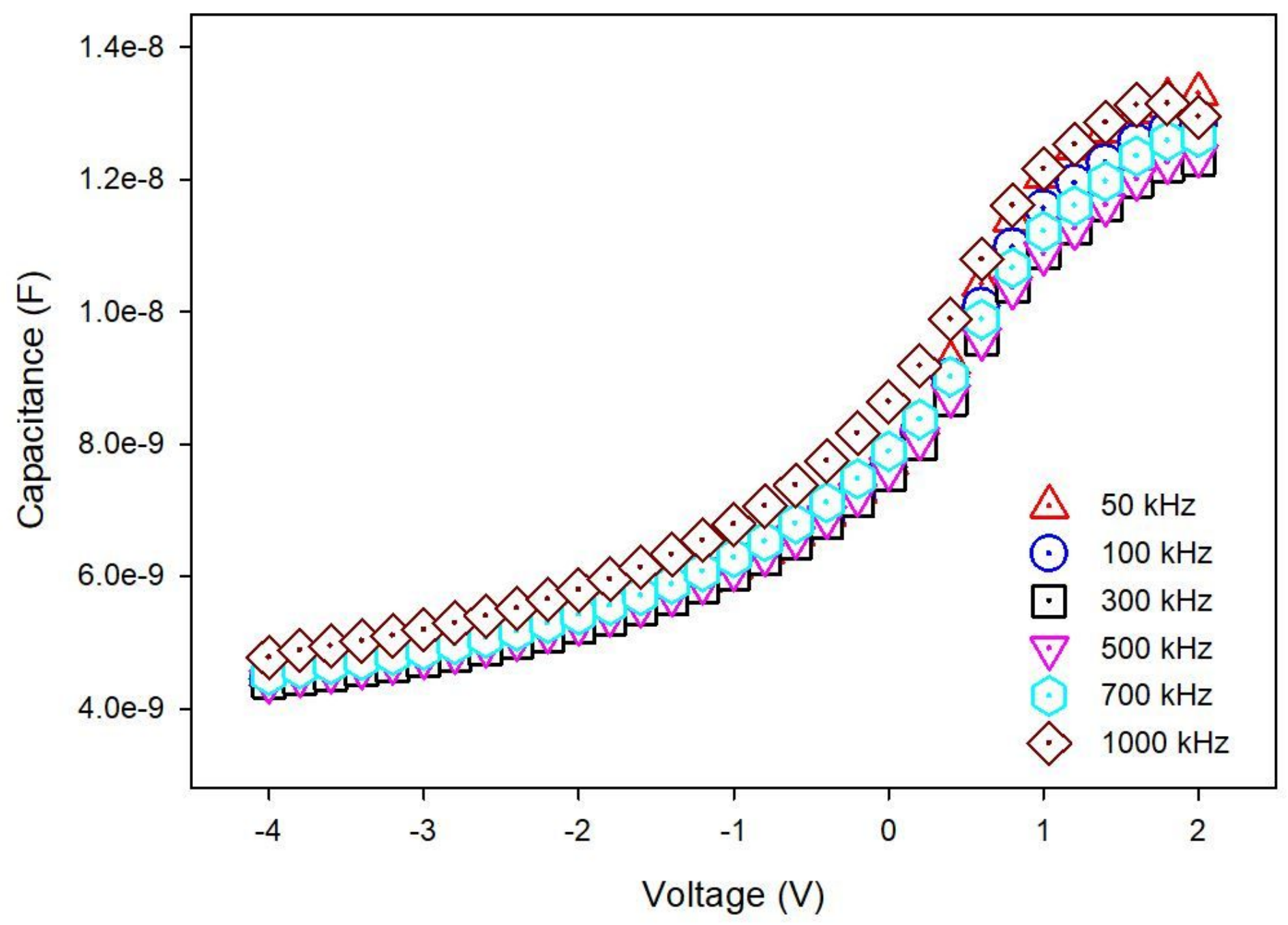

Figure 3

Capacitance-voltage graph of the $6 \mathrm{H}-\mathrm{SiC} / \mathrm{MEH}-\mathrm{PPV} / \mathrm{Al}$ diode 

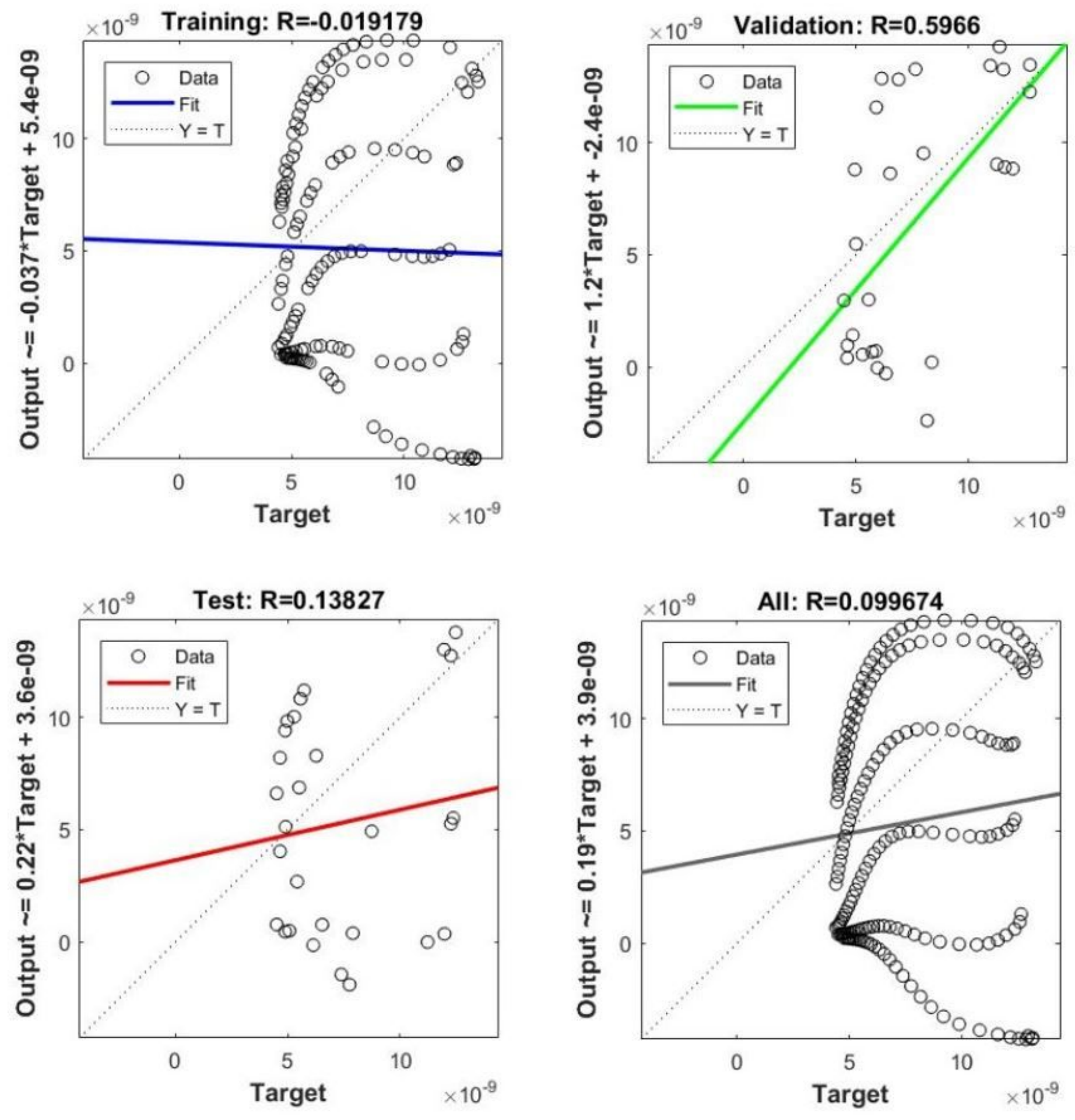

Figure 4

Performance of the ANN for all stages for neuron number 5 

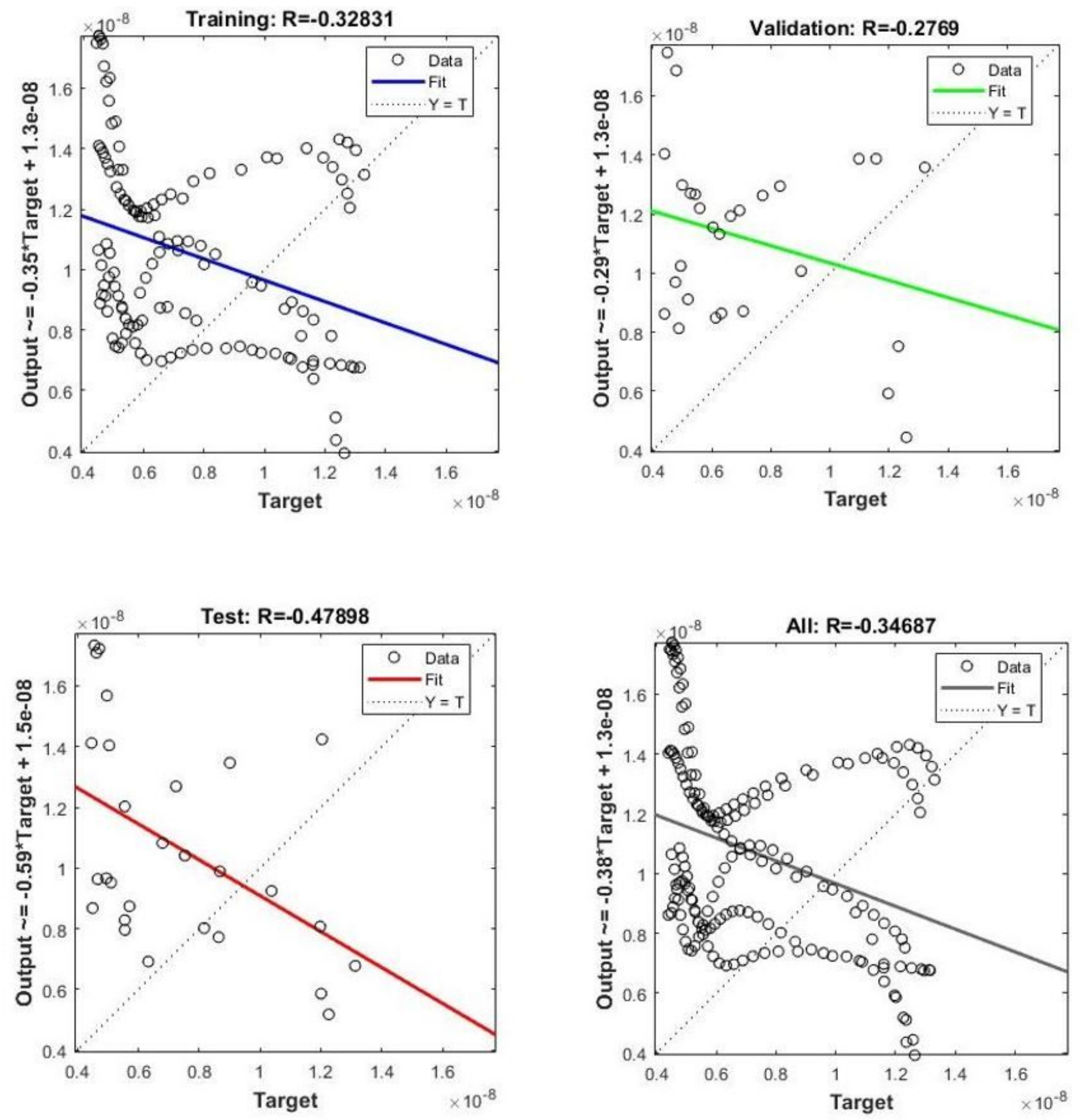

Figure 5

Performance of the ANN for all stages for neuron number 10 

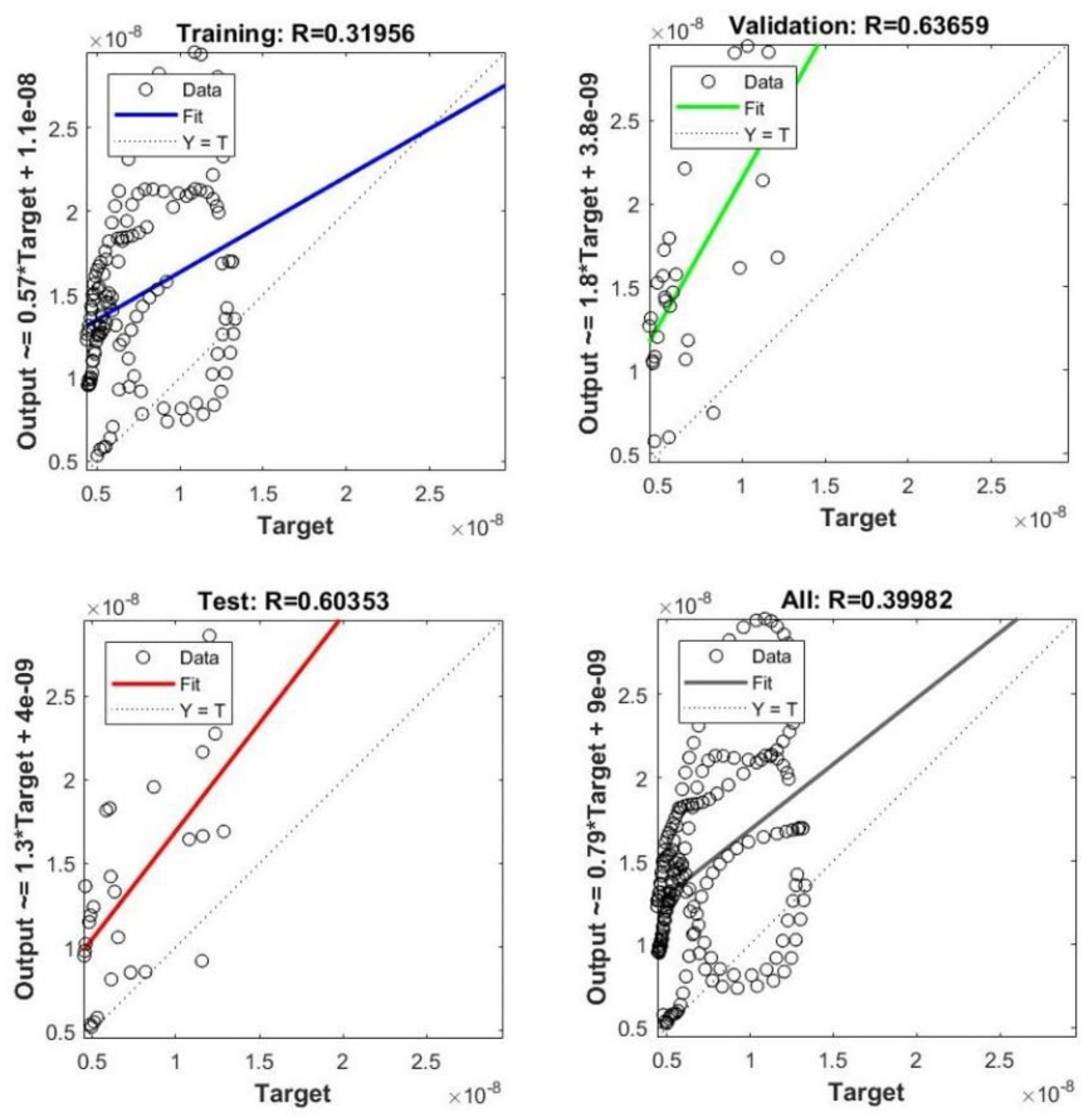

Figure 6

Performance of the ANN for all stages for neuron number 15 

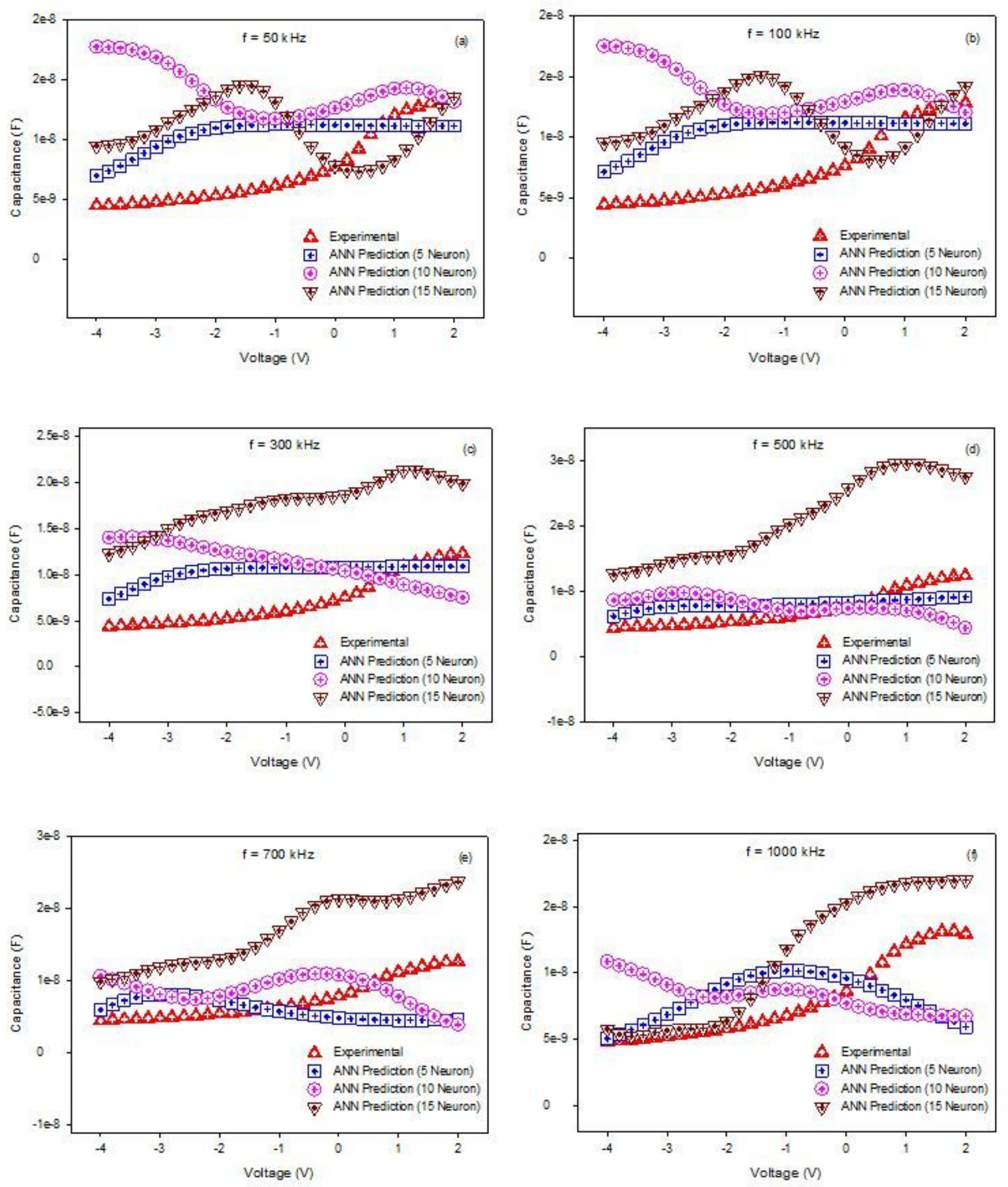

\section{Figure 7}

Experimental data and ANN predictions 

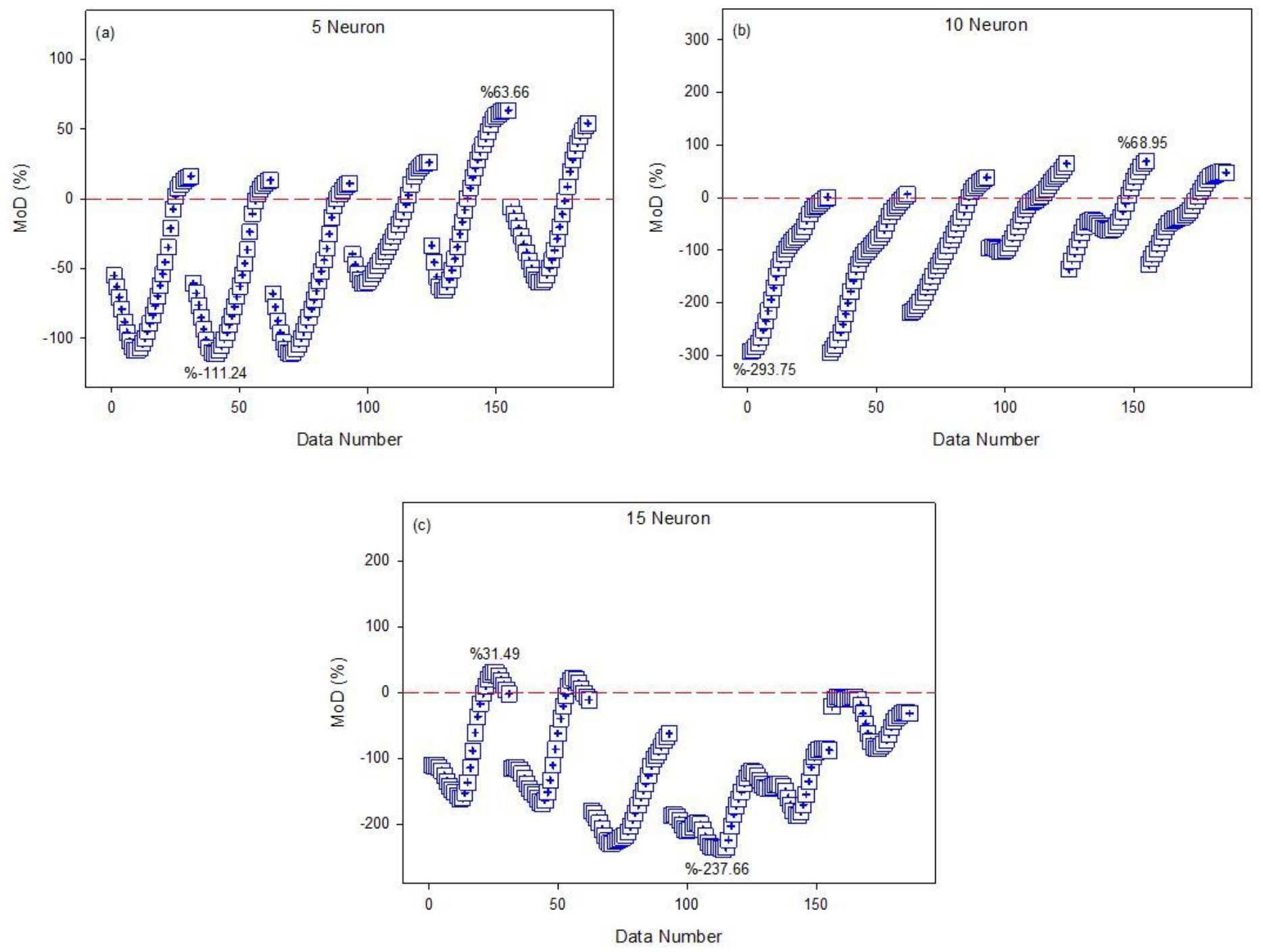

Figure 8

Error rates between the data obtained from the ANN model and the target values 

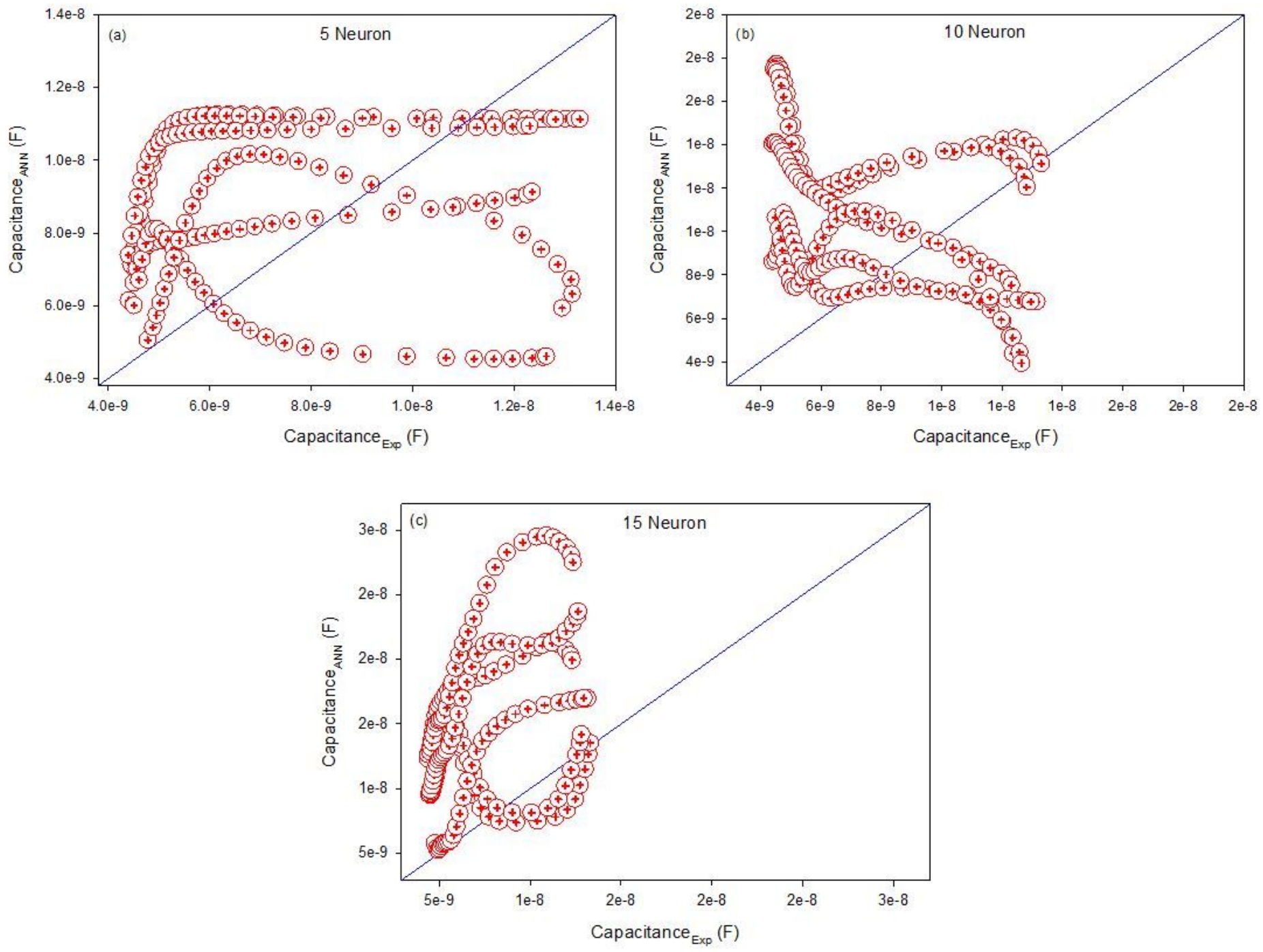

Figure 9

Predicted values and target values obtained from ANN model 